\title{
Dual-DNN Assisted Optimization for Efficient Resource Scheduling in NOMA-Enabled Satellite Systems
}

\author{
Anyue Wang, Lei Lei, Eva Lagunas, Symeon Chatzinotas, Björn Ottersten \\ Interdisciplinary Centre for Security, Reliability and Trust (SnT), University of Luxembourg, Luxembourg \\ Emails: \{anyue.wang; lei.lei; eva.lagunas; symeon.chatzinotas; bjorn.ottersten\}@uni.lu
}

\begin{abstract}
In this paper, we apply non-orthogonal multiple access (NOMA) in satellite systems to assist data transmission for services with latency constraints. We investigate a problem to minimize the transmission time by jointly optimizing power allocation and terminal-timeslot assignment for accomplishing a transmission task in NOMA-enabled satellite systems. The problem appears non-linear/non-convex with integer variables and can be equivalently reformulated in the format of mixedinteger convex programming (MICP). Conventional iterative methods may apply but at the expenses of high computational complexity in approaching the optimum or near-optimum. We propose a combined learning and optimization scheme to tackle the problem, where the primal MICP is decomposed into two learning-suited classification tasks and a power allocation problem. In the proposed scheme, the first learning task is to predict the integer variables while the second task is to guarantee the feasibility of the solutions. Numerical results show that the proposed algorithm outperforms benchmarks in terms of average computational time, transmission time performance, and feasibility guarantee.

Index Terms-Satellite communications, NOMA, deep learning, transmission time minimization, mixed-integer convex programming.
\end{abstract}

\section{INTRODUCTION}

Satellite communications have drawn growing attention owing to the advantages in providing wide coverage and high throughput [1]. However, due to the long distance between satellites and ground terminals, large transmission time restricts the performance of satellite systems, especially in the context of delivering services with latency restrictions [1], which motivates studies on resource optimization for satellite delay-constrained services [2]-[5]. The authors in [2] and [3] focused on delay-constrained resource allocation problems in satellite multi-beam and satellite-backhauling systems, respectively. In [4] and [5], the authors investigated completion time minimization problem in low-earth-orbit (LEO) satellite assisted uplink Internet-of-things networks. To embrace future trends of satellite communications, new applications, e.g., satellite-backhauling and content delivery, require more sophisticated design in terms of latency reduction.

Due to the capability of multiplexing more co-channel users and enhancing spectral efficiency than orthogonal multiple access (OMA), power-domain non-orthogonal multiple access (NOMA) has the potentials to further reduce delay for satellite systems. Satellite-NOMA has already proven its performance gains in capacity [6], outage performance [6], and offeredcapacity-to-requested-traffic ratio (OCTR) [7] compared to conventional satellite-OMA systems. However, resource allocation for transmission delay minimization in NOMA-enabled satellite systems has not been fully investigated yet. Delay is considered in the constraints of resource optimization in NOMA-based multi-beam satellite systems in [8]. In [5], the objective of the resource allocation problem is to minimize the completion time for LEO-satellite-assisted Internet of things systems. However, NOMA is applied in the terrestrial part but not in satellite transmission. It is still unknown how satelliteNOMA performs in transmission delay reduction compared to satellite-OMA systems. To the best of our knowledge, this is the first work to investigate resource optimization of transmission time minimization for NOMA-enabled satellite systems.

Another factor that influences delivering satellite delayconstrained services is the computational complexity of resource allocation algorithms. High-complexity algorithms may cause processing delays and thus fail to satisfy terminals' demands before deadlines [1]. A majority of resource allocation problems in satellite systems fall into the domain of constrained combinatorial optimization [2]-[5], [7], [9], [10]. Conventional algorithms for solving such problems may consume a large amount of computational efforts and time in attaining the optimum or near-optimum, and with limited capability to well balance optimality and computational complexity [11]. In comparison to model-based optimization, datadriven learning techniques can exploit useful information from empirical data first, and approximate optimal decisions with less computational complexity [12]

Applying deep learning (DL) techniques to satellite systems has been studied from various aspects, e.g., predicting optimal decoding order for NOMA-based satellite systems [13], assisting beam-timeslot scheduling for beam hopping satellite systems [14], compensating for the non-linear distortion at receivers in LEO satellite systems [15], etc. Applying DL to address constrained combinatorial problems is, however, studied to a limited extent in the literature. In addition, directly applying end-to-end learning may not achieve desired performance due to feasibility issues [16]. The challenges of 
designing a DL-based solution lie in how to apply DL to obtain a solution of constrained combinatorial optimization with low complexity, and at the meantime, achieve satisfactory approximating performance and guarantee a feasible solution by meeting all the constraints.

In this paper, a DL-assisted approach is designed to minimize transmission time in NOMA-enabled satellite systems. The main contributions are summarized as follows.

- We formulate a mixed-integer non-linear programming (MINLP) problem to minimize transmission time via power allocation and timeslot-terminal assignment in satellite NOMA systems. With the identified convexity, we reformulate the primal problem as mixed-integer convex programming (MICP), whose optimum or near optimum can be achieved by applying mature optimization methods in spite of high complexity.

- As mentioned above, simply applying end-to-end learning may not provide a near-optimal solution and guarantee the feasibility in terminal-timeslot-power allocation for the considered MICP. We investigate a combined scheme with data-driven learning and model-based optimization approaches. To properly tackle the combinatorial optimization problem by DL, we extract two learning tasks from the primal MICP, i.e., classifying binary terminaltimeslot allocation and identifying its feasibility in power allocation. Both are classification-like tasks and suited to be learned by deep neural networks (DNN). With the obtained terminal-timeslot allocation, the remaining power allocation is optimally and efficiently solved by convex optimization.

- The numerical results demonstrate the superiority of the proposed algorithm in computational time, near optimality, and feasibility guarantee, compared with state-of-theart optimal and suboptimal solutions.

\section{System Model}

We consider resource allocation for forward-link transmission in a geostationary earth orbit (GEO) satellite system. In the system, four-color frequency-reuse pattern is implemented, where the bandwidth is equally segmented into two portions and each portion makes use of vertical and horizontal polarization. Each color is occupied by one of the neighboring four beams, such that any two adjacent beams can access to orthogonal resources [1]. Hence, inter-beam interference has limited impacts on other beams and can be viewed as fixed. Resource optimization can be therefore decoupled into each beam. In this paper, we focus on the algorithmic design of resource allocation for the single-beam scenario. For practical implementation, the proposed algorithm can be executed parallelly in the resource manager at the gateway side for multiple beams.

We consider that the satellite system provides services to $K$ fixed ground terminals within the beam. Terminals' requested demands need to be satisfied by allocating power and scheduling timeslots to terminals before the deadline. We define that the number of available timeslots is up to
$T$ timeslots. In conventional OMA satellite systems, each timeslot can only be assigned to one terminal. In NOMAenabled satellite systems, however, more than one terminal is allowed to access each timeslot. The higher efficiency of resource utilization in NOMA may enable less duration in delivering deadline-constrained services for satellite systems compared to conventional OMA schemes.

According to the basis of NOMA, terminals' signals are superimposed at the gateway and transmitted to the satellite then to the corresponding ground terminals. After receiving the signals, the terminal performs successive interference cancellation (SIC) to decode and eliminate part of the cochannel interference. We denote the channel gain of terminal $k$ as $G_{k}$, which is derived by,

$$
G_{k}=G_{k}^{\mathrm{Tx}} L_{k} G_{k}^{\mathrm{Rx}}
$$

Here, $G_{k}^{\mathrm{Tx}}$ denotes the satellite transmit antenna gain depending on the off-axis angle between terminal $k$ and the corresponding beam center. $G_{k}^{\mathrm{Rx}}$ is the receive antenna gain of terminal $k$. $L_{k}$ represents the free-space path loss from the satellite to the $k$-th terminal. The path loss is computed by $L_{k}=\left(\frac{c}{4 \pi f d_{k}}\right)^{2}$, where $c, f$, and $d_{k}$ denote the light speed, the frequency, and the distance between the satellite and terminal $k$, respectively. In a GEO satellite system with fixed ground terminals, the channel gains stay unchanged with large coherence time. The decoding order is determined based on the descending order of the channel gains. For presentation simplicity, we define that terminals' indices keep the same with the decoding order, i.e., $G_{1}>G_{2}>\cdots>G_{K}$. The signal-tointerference-plus-noise ratio (SINR) of terminal $k$ at timeslot $t$ is expressed as:

$$
\mathrm{SINR}_{k t}=\frac{G_{k} P_{k t}}{\sum_{j=1}^{k-1} G_{k} P_{j t}+I_{k}+\sigma^{2}}
$$

where $P_{k t}$ denotes the transmit power of terminal $k$ at timeslot $t . I_{k}$ is the empirical expectation of the inter-beam interference to terminal $k . \sigma^{2}$ is the noise power. In (2), terminal $k$ is unable to decode terminal $j$ 's signal when $j<k$, and terminal $j$ 's signal is thus treated as interference at terminal $k$ 's receiver. For $j>k$, terminal $k$ performs SIC to decode and remove $j$ 's signal such that the inter-terminal interference can be reduced. Note that, if $P_{k t}=0, \mathrm{SINR}_{k t}=0$ and other terminals will not suffer the co-channel interference from the $k$-th terminal. The allocated capacity for terminal $k$ at timeslot $t$ can be derived as

$$
C_{k t}=W \log \left(1+\operatorname{SINR}_{k t}\right),
$$

where $W$ is the occupied bandwidth. Thus, the achievable offered capacity of terminal $k$ is derived as

$$
C_{k}=\sum_{t=1}^{T} C_{k t}
$$




\section{Problem Formulation AND ANALYsis}

We formulate a resource optimization problem to minimize the transmission time of accomplishing a task for delivering requested services to ground terminals. The variables are defined as follows:

$0 \leq P_{k t} \leq P_{\max }$, transmit power of terminal $k$ at timeslot $t$ $a_{k t}=\left\{\begin{array}{l}1, \text { terminal } k \text { is scheduled at timeslot } t \\ 0, \text { otherwise }\end{array}\right.$ $b_{t}= \begin{cases}1, & \text { timeslot } t \text { is assigned by any terminal } \\ 0, & \text { otherwise }\end{cases}$

We define vectors $\mathbf{a}, \mathbf{b}$, and $\mathbf{P}$ to collect all the $a_{k t}, b_{t}, P_{k t}$ variables, respectively. The problem is formulated as,

$$
\begin{aligned}
\mathcal{P}_{1}: & \min _{\mathbf{a}, \mathbf{b}, \mathbf{P}} \sum_{t=1}^{T} b_{t} \tau+\max _{k}\left\{\frac{d_{k}+d^{\mathrm{GW}}}{c}\right\} \\
\text { s.t. } & C_{k} \geq D_{k}, \forall k=1, \ldots, K, \\
& \left(C_{k}-D_{k}\right)^{2} \leq \Delta, \forall k=1, \ldots, K, \\
& \sum_{k=1}^{K} P_{k t} \leq P_{\max }, \forall t=1, \ldots, T, \\
& \sum_{k=1}^{K} a_{k t} \leq \bar{K} b_{t}, \forall t=1, \ldots, T, \\
& b_{t} \geq b_{t+1}, \forall t=1, \ldots, T-1, \\
& P_{k t} \leq P_{\max } a_{k t}, \forall k=1, \ldots, K, \forall t=1, \ldots, T .
\end{aligned}
$$

Here, $\tau$ denotes the duration of one timeslot and $d^{\mathrm{GW}}$ denotes the distance between the gateway and the satellite. In the objective, the transmission time includes completion time, defined as the total duration of all the occupied timeslots, and transmission delay, defined as the maximum propagation duration among terminals. To avoid unnecessary delay, constraints (5f) restrict that no idle timeslot appears before the transmission task is accomplished. Once the $t$-th timeslot is not assigned to any terminals, the timeslots after $t$ will be no longer scheduled. Constraints (5b) denote that each terminal's demand $D_{k}$ must be satisfied within at most $T$ timeslots. In (5c), the difference between offered capacity and requested demands of each terminal should be smaller than $\Delta$ to reduce unused capacity. In (5d), the total consumed power at each timeslot is no more than the beam power budget $P_{\max }$. Constraints $(5 \mathrm{e})$ confine the number of scheduled terminals at each timeslot no larger than $\bar{K}$. Constraints (5e) also connect a with $\mathbf{b}$. That is $\sum_{k=1}^{K} a_{k t}=0$ if $b_{t}=0$. Note that the solution $\sum_{k=1}^{K} a_{k t}=0$ and $b_{t}=1$ is clearly not the optimal and thus is excluded from the optimum. Constraints $(5 \mathrm{~g})$ confine the dependence between $\mathbf{P}$ and $\mathbf{a}$, where $P_{k t}=0$ if $a_{k t}=0$, and $P_{k t} \leq P_{\max }$ if $a_{k t}=1$.

We observe that $\mathcal{P}_{1}$ is identified as an MINLP due to the presence of binary variables $\mathbf{a}, \mathbf{b}$, and non-linear function $C_{k t}$ in (2) and (3) [17]. To reveal the convexity of the original problem, we derive $P_{k t}$ as the function of capacity
$C_{k t}$ based on (2) and (3) via a substituting procedure [11]. Then $\sum_{k=1}^{K} P_{k t}$ reads:

$$
\begin{aligned}
\sum_{k=1}^{K} P_{k t}= & \sum_{k=1}^{K}\left(\frac{I_{k}+\sigma^{2}}{G_{k}}-\frac{I_{k-1}+\sigma^{2}}{G_{k-1}}\right) \exp \left(\frac{\sum_{j \geq k} C_{j t}}{W}\right) \\
& -\frac{I_{K}+\sigma^{2}}{G_{K}}
\end{aligned}
$$

where we define that $\frac{I_{0}+\sigma^{2}}{G_{0}}=0$. Substituting (6) into $\mathcal{P}_{1}$, the original problem is equivalently reformulated as,

$$
\begin{aligned}
& \mathcal{P}_{2}: \min _{\mathbf{a}, \mathbf{b}, \mathbf{C}} \sum_{t=1}^{T} b_{t} \tau+\max _{k}\left\{\frac{d_{k}+d^{\mathrm{GW}}}{c}\right\} \\
& \text { s.t. }(5 \mathrm{~b}),(5 \mathrm{c}),(5 \mathrm{e}),(5 \mathrm{f}), \\
& \sum_{k=1}^{K}\left(\frac{I_{k}+\sigma^{2}}{G_{k}}-\frac{I_{k-1}+\sigma^{2}}{G_{k-1}}\right) \exp \left(\frac{\sum_{j \geq k} C_{j t}}{W}\right) \\
& \quad-\frac{I_{K}+\sigma^{2}}{G_{K}} \leq P_{\max }, \forall t=1, \ldots, T, \\
& C_{k t} \leq C_{\max } a_{k t}, \forall k=1, \ldots, K, \forall t=1, \ldots, T,
\end{aligned}
$$

where vector $\mathbf{C}$ collects all the capacity variables $C_{k t}$. The optimization variables are $\mathbf{a}, \mathbf{b}$, and $\mathbf{C}$. Similar to $(5 \mathrm{~g})$, we confine the relationships between $\mathbf{C}$ and $\mathbf{a}$ in $(7 \mathrm{~d})$, where $C_{\max }>\max _{k, t}\left\{C_{k t}\right\}$. That is, $C_{k t}=0$ if $a_{k t}=0$, and $C_{k t} \leq C_{\max }$ if $a_{k t}=1$. Note that since $\mathcal{P}_{1}$ and $\mathcal{P}_{2}$ are equivalent, the solution of $\mathbf{P}$ can be computed by the expression of $\mathbf{C}$ based on the substituting procedure after solving $\mathcal{P}_{2}$.

The expression of $(7 \mathrm{c})$ is the sum of exponential functions, which is convex [17]. More specifically, we recognize that the convex constraints (7c) are in fact in the format of nonsymmetric exponential cones. Besides, constraints (5c) are in the convex quadratic format. Thus $\mathcal{P}_{2}$ is identified as an MICP problem [18]. On the one hand, optimal or near-optimal solutions of MICP problems can be obtained by conventional branch-and-bound or outer-approximation methods [19]. Some state-of-the-art solvers, e.g., MOSEK [20], can also apply. Nevertheless, the intrinsic difficulties in discrete optimization lead to exponentially increased computational complexity and time, which are not affordable in practical scenarios. With deadline constraints, the applicability of these approaches in satellite systems may be inevitably cause the failure of accomplishing a transmission task.

On the other hand, DL has already proven satisfactory performance in several learning tasks, e.g., classification and regression, where a mapping from training inputs to outputs is learned [21]. However, DL may not be directly applicable to complicated mixed-integer optimization problems like $\mathcal{P}_{2}$. Direct end-to-end learning, for example, does not perform well in the prediction of optimal integer solutions [16] since imperfect prediction will lead to dissatisfaction of some constraints and thus the infeasibility issues. It is essential to identify whether the predicted solution is feasible or not and to obtain a nearoptimal feasible solution efficiently. How to design a DL-based approach to appropriately address $\mathcal{P}_{2}$ is non-trivial. 


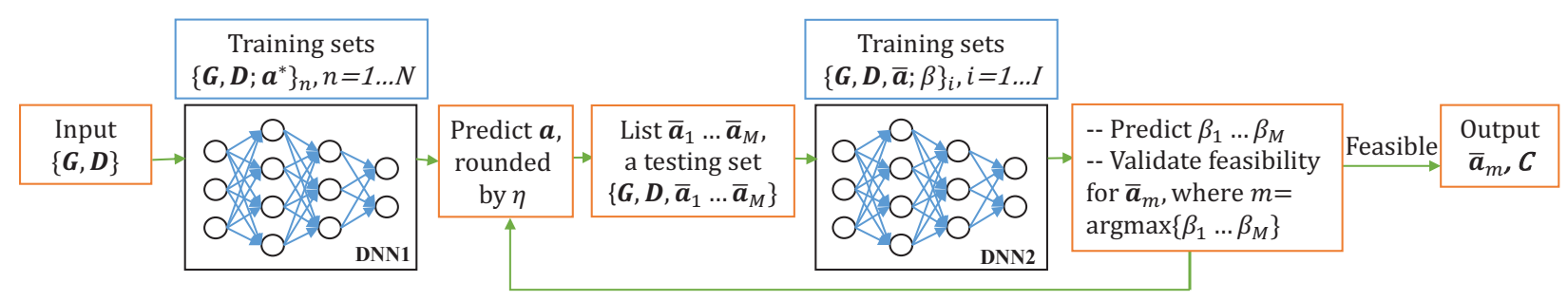

Power/rate allocation is infeasible, $\eta=\eta-\theta$

Fig. 1. Illustration of the proposed DDCO

\section{The Proposed DNN-BASED APPROACH}

In this section, we propose a hybrid solution combined with dual DNNs and convex optimization (DDCO) to tackle $\mathcal{P}_{2}$. The idea is that we rely on fast prediction in dual DNNs to tackle the most difficult and time-consuming part in MICP, i.e., optimizing discrete variables $\mathbf{a}$ and $\mathbf{b}$, instead of performing iterative searching algorithms. The remaining power (or capacity) allocation can be solved by efficient convex optimization approaches. Overall, DDCO is expected to reap advantages from learning and optimization, and thus to enable an efficient, feasible, and near-optimal solution. The procedure is illustrated in Fig. 1.

In DDCO, we extract two classification-like tasks from $\mathcal{P}_{2}$, and let dual DNNs train and learn from optimal labels to provide a promising terminal-timeslot allocation with identified feasibility. The $1-\mathrm{st} \mathrm{DNN}$ is used to predict the binary elements in a, i.e., learning the mapping from the input of channel-gain vector $\mathbf{G}=\left[G_{1}, \ldots, G_{K}\right]$ and demand vector $\mathbf{D}=\left[D_{1}, \ldots, D_{K}\right]$ to optimal terminal-timeslot allocation $\mathbf{a}^{*}$. We use a tuple $\left\{\mathbf{G}, \mathbf{D} ; \mathbf{a}^{*}\right\}_{n}$ to represent the $n$-th trainingtesting set, where the input vectors $\mathbf{G}$ and $\mathbf{D}$ are generated from the $n$-th realization in an emulator, and the corresponding optimal label $\mathbf{a}^{*}$ can be obtained by solving $\mathcal{P}_{2}$ offline. The predicted a from the 1-st DNN may not necessarily be binary, a threshold $\eta$ is then introduced to round the fractional elements to binary.

Ideally, the predicted a should lead to a feasible terminaltimeslot allocation. That is, satisfying $\sum_{t=1}^{T} a_{k t} \geq 1, \forall k=$ $1, \ldots, K$, i.e., each terminal is scheduled to at least one timeslot to satisfy constraints (5b), and no violation for constraints (5e) and (5f). However, these feasibility conditions cannot be always guaranteed because of DL's imperfect prediction. To deal with this issue, we perform a post-process for the predicted a to list $M$ feasible and promising terminal-timeslot allocation $\overline{\mathbf{a}}_{1}, \ldots, \overline{\mathbf{a}}_{M}$. We use an example with $K=4$, $T=4, \bar{K}=2$ to illustrate the process. After rounding, the predicted a reads, $[\underbrace{1,1,1,0}, \underbrace{1,0,1,1}, \underbrace{0,0,0,0}, \underbrace{1,0,1,0}]$ $\underbrace{1,1,1,}_{a_{11}, \ldots, a_{41}} \underbrace{1,0,1,}_{a_{12}, \ldots, a_{42}} \underbrace{0,0,0,}_{a_{13}, \ldots, a_{43}}$ This allocation consumes 3 timeslots but the completion time is 4 timeslots, which violates constraints (5f), and schedules 3 terminals on the first two timeslots, which violates constraints (5e). To satisfy (5f), we move the terminals scheduled at timeslot 4 to timeslot 3 and leave timeslot 4 idle. To meet (5e), we select $\bar{K}=2$ terminals from 3 for the 1 -st and 2-nd timeslots, giving 9 combinations $\left(\left(\begin{array}{l}3 \\ 2\end{array}\right) \times\left(\begin{array}{l}3 \\ 2\end{array}\right)\right)$ in total. Filtered by constraints $\sum_{t=1}^{T} a_{k t} \geq 1, \forall k=1, \ldots, K, 4$ out of 9 feasible terminal-timeslot allocations $\overline{\mathbf{a}}_{1}, \ldots, \overline{\mathbf{a}}_{4}$ are generated.

A follow-up question is that whether a feasible power (or capacity) allocation (satisfying constraints (5b), (5c), (7c), and (7d)) exists under these feasible terminal-timeslot allocations. One may notice that once $\mathbf{a}$ is fixed in $\mathcal{P}_{2}$, then $\mathbf{b}$ is determined. The remaining power (or capacity) optimization problem becomes a decision-version problem, that is providing a yes-or-no answer to the existence of feasible power (or capacity) allocation under the certain $\mathbf{a}$ and $\mathbf{b}$. This feasibilitycheck problem is presented as,

$$
\mathcal{P}_{3} \text { : Find C, s.t. (5b), (5c), (7c), (7d), }
$$

which amounts to solving a convex optimization problem.

In general, the complexity for answering a problem's feasibility is of the same magnitude as solving the corresponding optimization problem. Thus in order to identify at least one feasible solution from $\overline{\mathbf{a}}_{1}, \ldots, \overline{\mathbf{a}}_{M}$, in the worst case, $M$ convex problems have to be solved, which may not be computationally affordable when $M$ is large. We train the 2nd DNN to enable a quick feasibility check for $\overline{\mathbf{a}}_{1}, \ldots, \overline{\mathbf{a}}_{M}$. The training sets are organized in tuples $\{\mathbf{G}, \mathbf{D}, \overline{\mathbf{a}} ; \beta\}_{i}, i=$ $1, \ldots, I$, where $\overline{\mathbf{a}}$ is a terminal-timeslot allocation, and optimal label $\beta$, obtained by solving $\mathcal{P}_{3}$ via convex optimization, stands for the corresponding feasibility $(\beta=1)$ or infeasibility $(\beta=0)$ of $\overline{\mathbf{a}}$. A mapping from inputs $\mathbf{G}, \mathbf{D}, \overline{\mathbf{a}}$ to optimal label $\beta$ is learned. In the testing phase, the value of the $m$ th output node, $\beta_{m}$, refers to the probability of a feasible power (or capacity) solution existing for $\overline{\mathbf{a}}_{m}$. We select the terminal-timeslot allocation with the highest probability, say the $m$-th element $\beta_{m}=\max \left\{\beta_{1}, \ldots, \beta_{M}\right\}$, and solve the convex optimization problem under $\overline{\mathbf{a}}_{m}$ in order to validate the feasibility of power (or capacity) allocation to against DNN's imperfect prediction. If no feasible power exists, we reduce threshold $\eta$ by step $\theta$ to involve more feasible terminal-timeslot allocations to set $\left\{\overline{\mathbf{a}}_{1}, \ldots, \overline{\mathbf{a}}_{M}\right\}$, otherwise, DDCO outputs the feasible terminal-timeslot allocation $\overline{\mathbf{a}}_{M}$ and corresponding capacity (or power) allocation $\mathbf{C}$ (or $\mathbf{P}$ ).

\section{Performance Evaluation}

In this section, we evaluate the performance of the proposed DDCO. The parameter settings are summarized in Table I. 
TABLE I

SiMUlation PARAMETERS

\begin{tabular}{c|c}
\hline Parameter & Value \\
\hline Frequency & $20 \mathrm{GHz}(\mathrm{Ka}$ band $)$ \\
Bandwidth $(W)$ & $250 \mathrm{MHz}$ \\
Satellite location & $13^{\circ} \mathrm{E}$ \\
Satellite height $\left(d^{\mathrm{GW}}, d_{k}\right)$ & $35,786 \mathrm{~km}$ \\
Beam radiation pattern & provided by ESA [22] \\
Receive antenna gain & $42.1 \mathrm{dBi}$ \\
Noise power $\left(\sigma^{2}\right)$ & $-126.47 \mathrm{dBW}$ \\
Maximum transmit power $\left(P_{\max }\right)$ & $20 \mathrm{dBW}$ \\
Number of time slots $(T)$ & 100 \\
Maximum multiplexed users $(\bar{K})$ & 2 \\
Duration of each timeslot $(\tau)$ & $1 \mathrm{~ms}$ \\
Demand $\left(D_{k}\right)$ & 325 to $750 \mathrm{Mbps}$ \\
Training samples $(N)$ & 6000 \\
Testing samples $(I)$ & 1000
\end{tabular}

Both DNNs are implemented in TensorFlow, where ReLu, Mean Square Error (MSE), and Adam algorithm are adopted as the activation function, loss function, and optimizer, respectively. The inputs $\mathbf{G}$ and $\mathbf{D}$ are generated from the adopted emulator based on the parameter settings in TABLE I, where the beam radiation pattern is provided by European Space Agency (ESA) in the context of [22]. We set the following approaches as benchmarks:

- Opt: The optimal solution is obtained by solving $\mathcal{P}_{2}$ with Mosek solver [20], which is capable of tackling MICP by branch-and-cut approaches.

- BW-FTPC [23]: Terminals are grouped on the basis of best-worst $(\mathrm{BW})$ pairing and power allocation is based on fractional transmit power control (FTPC) rule.

- SM-CP [24]: Terminal-timeslot assignment is iteratively updated by swap-matching (SM) approach. For each iteration, a conic programming $(\mathrm{CP})$ of capacity allocation should be solved.

- SD: The single-DNN (SD) scheme follows the procedure of DDCO but without using the 2-nd DNN, instead, adopting convex optimization to verify the capacity (or power) feasibility for $\overline{\mathbf{a}}_{1}, \ldots, \overline{\mathbf{a}}_{M}$.

- OMA: Each timeslot can be only scheduled to one terminal, i.e., $\bar{K}=1$.

TABLE II

Average Computational Time (In Seconds) $(\eta=0.2)$

\begin{tabular}{c|c|c|c|c|c}
\hline$K$ & Opt & BW-FTPC & SM-CP & SD & DDCO \\
\hline 4 & 1.33 & 0.12 & 0.64 & 1.79 & 0.325 \\
\hline 6 & 5.17 & 0.13 & 3.51 & 10.32 & 0.577 \\
\hline 8 & 38.23 & 0.16 & 6.74 & 123.28 & 0.698 \\
\hline 10 & 798.4 & 0.17 & 9.38 & 146.05 & 0.842 \\
\hline
\end{tabular}

Firstly, in TABLE II, we compare the average computational time among Opt, BW-FTPC, SM-CP, SD, and DDCO. In the optimal algorithm, the computation time increases exponentially as the problem's scale increases. In comparison, the computational time in BW-FTPC and DDCO is significantly reduced to millisecond level while SM-CP consumes time in second level. SD consumes much longer time than DDCO since the complexity for solving up to $M$ convex problems in SD remains considerable. With $K$ increases, the proposed
DDCO is capable of providing an overall computationally efficient solution.

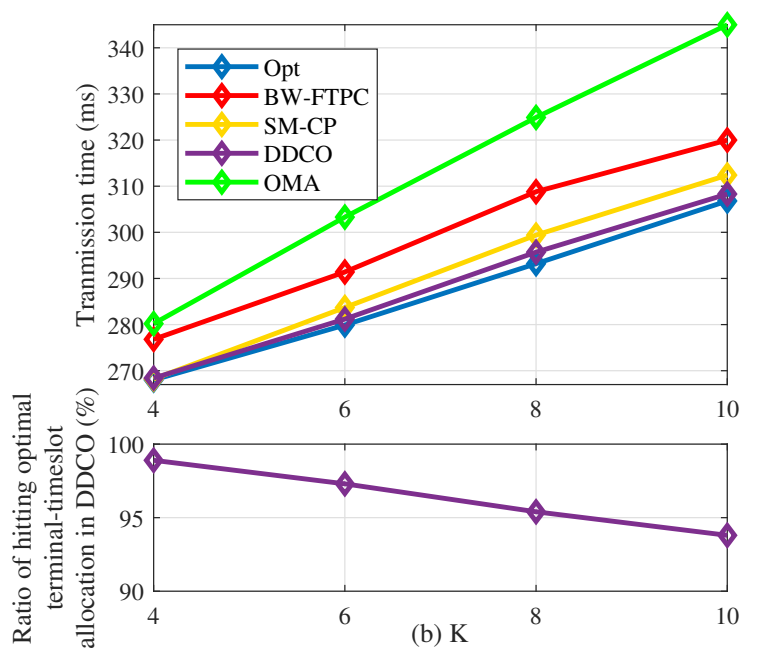

Fig. 2. (a) Minimized transmission time with respect to $K(\eta=0.14$ in DDCO); (b) DDCO in hitting optimal terminal-timeslot allocation $\mathbf{a}^{*}$.

Secondly, in Fig. 2, we evaluate DDCO's performance in approximating the optimum. In Fig. 2(a), we compare the objective values, i.e., minimized transmission time, in Opt, BW-FTPC, SM-CP, DDCO, and OMA. In general, the gap between optimal and suboptimal solutions increases significantly as the number of terminals grows, whereas the gap between DDCO and the optimum keeps less than $5 \%$ in average. The main reason for this near optimality is that, when $M$ feasible terminal-timeslot allocations are generated for the 2-nd DNN, DDCO has strong capability to retain the optimal allocation $\mathbf{a}^{*}$ in the candidate set, i.e., $\mathbf{a}^{*} \in\left\{\overline{\mathbf{a}}_{1}, \ldots, \overline{\mathbf{a}}_{M}\right\}$. This is verified in Fig. 2(b), where DDCO hits optimal $\mathbf{a}^{*}$ in more than $95 \%$ testing sets in average. Recall that by design, $\overline{\mathbf{a}}_{1}, \ldots, \overline{\mathbf{a}}_{M}$ have the same objective value. As a consequence, even though DDCO's outputted allocation $\overline{\mathbf{a}}_{m}$ may not be necessarily as same as the optimal $\mathbf{a}^{*}$, the optimal objective value can be achieved when $\mathbf{a}^{*} \in\left\{\overline{\mathbf{a}}_{1}, \ldots, \overline{\mathbf{a}}_{M}\right\}$ holds.

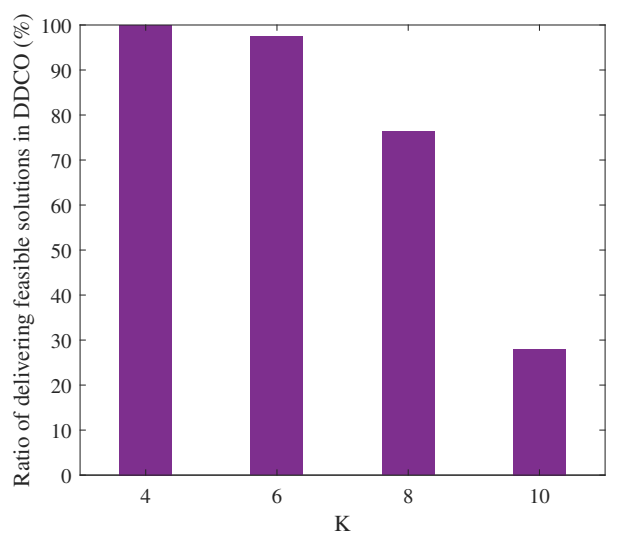

Fig. 3. DDCO in delivering feasible solutions with respect to $K(\eta=0.2)$.

Thirdly, in Fig. 3, we demonstrate DDCO's performance in delivering feasible solutions, and show the significance of choosing an appropriate threshold $\eta$. In Fig. 3, the adopted 


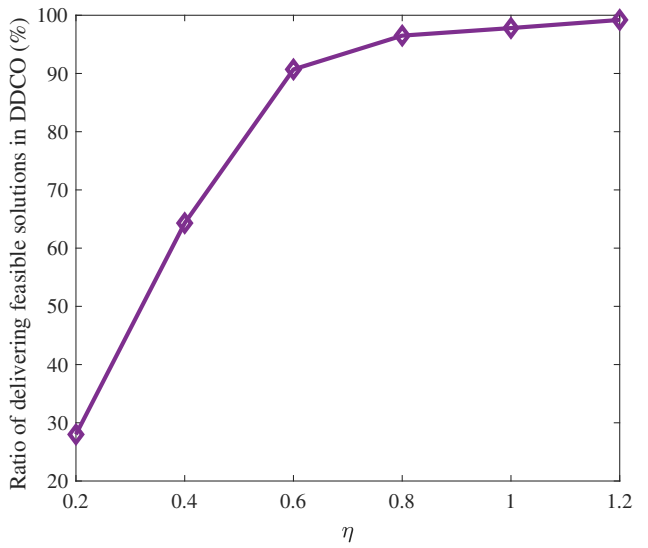

Fig. 4. DDCO in delivering feasible solutions with respect to threshold $\eta$ $(K=10)$.

threshold $\eta=0.2$ is suited for 4-terminal cases, i.e., leading to a feasible terminal-timeslot and capacity (or power) solutions in $99 \%$ instances. In contrast, the same threshold for 10terminal cases fails, leading to infeasible solutions in most of the instances. Next, in Fig. 4, we decrease $\eta$ for 10-terminal cases, and observe the significant performance improvement. The reason is that when $\eta$ decreases in the 1-st DNN, the fractional elements in the predicted vector a are prone to be rounded to 1 , then it results in more allocations in set $\left\{\overline{\mathbf{a}}_{1}, \ldots, \overline{\mathbf{a}}_{M}\right\}$. Smaller $\eta$ and larger $M$ in fact increase the probability of DDCO in obtaining a feasible capacity (or power) allocation. The results also necessitate the thresholdadjustment procedure $(\eta=\eta-\theta)$ in DDCO.

\section{CONCLUSION}

In this paper, we have formulated a problem to minimize transmission time by optimizing transmit power and terminaltimeslot scheduling in an NOMA-enabled satellite system. We have investigated how to apply DL to address a typical combinatorial optimization problem. The primal problem has been reformulated in the format of MICP, such that the optimum can be obtained by conventional iterative approaches. Considering its inherent difficulty and high complexity, we have proposed a hybrid solution combining data-driven learning and modelbased optimization to provide an efficient, feasible, and nearoptimal solution.

\section{ACKNOWLEDGMENT}

The work has been funded by the Fonds National de la Recherch (FNR) CORE project ROSETTA (11632107) and the European Research Council (ERC) Project AGNOSTIC under Grant 742648.

\section{REFERENCES}

[1] O. Kodheli et al., "Satellite communications in the new space era: A survey and future challenges," IEEE Communications Surveys \& Tutorials, 2020.

[2] X. Zhong, Y. He, H. Yin, J. Wang, and Z. Du, "Joint power and timeslot allocation based on delay priority for multi-beam satellite downlinks," in 2017 International Conference on Progress in Informatics and Computing (PIC). IEEE, 2017, pp. 389-393.
[3] Z. Ji, S. Cao, S. Wu, and W. Wang, "Delay-aware satellite-terrestrial backhauling for heterogeneous small cell networks," IEEE Access, vol. 8, pp. 112 190-112202, 2020.

[4] C. Liu, R. Chai, and Q. Chen, "IoT gateway association and data scheduling for delay optimization in LEO satellite systems," in 2020 IEEE/CIC International Conference on Communications in China (ICCC). IEEE, 2020, pp. 993-998.

[5] Z. Gao, A. Liu, C. Han, and X. Liang, "Max completion time optimization for internet of things in LEO satellite-terrestrial integrated networks," IEEE Internet of Things Journal, 2021.

[6] X. Yan, H. Xiao, C.-X. Wang, K. An, A. T. Chronopoulos, and G. Zheng, "Performance analysis of NOMA-based land mobile satellite networks," IEEE Access, vol. 6, pp. 31 327-31339, 2018.

[7] A. Wang, L. Lei, E. Lagunas, A. Perez-Neira, S. Chatzinotas, and B. Ottersten, "Noma-enabled multi-beam satellite systems: Joint optimization to overcome offered-requested data mismatches," IEEE Transactions on Vehicular Technology, 2020.

[8] X. Liu, X. B. Zhai, W. Lu, and C. Wu, "QoS-guarantee resource allocation for multibeam satellite industrial internet of things with NOMA," IEEE Transactions on Industrial Informatics, vol. 17, no. 3, pp. 2052-2061, 2019.

[9] S. Kisseleff, E. Lagunas, T. S. Abdu, S. Chatzinotas, and B. Ottersten, "Radio resource management techniques for multibeam satellite systems," IEEE Communications Letters, pp. 1-1, 2020.

[10] Y. Xu, R. Q. Hu, and G. Li, "Robust energy-efficient maximization for cognitive NOMA networks under channel uncertainties," IEEE Internet of Things Journal, vol. 7, no. 9, pp. 8318-8330, 2020.

[11] L. Lei, D. Yuan, C. K. Ho, and S. Sun, "Power and channel allocation for non-orthogonal multiple access in 5G systems: Tractability and computation," IEEE Transactions on Wireless Communications, vol. 15 , no. 12, pp. 8580-8594, 2016.

[12] M. Chen, U. Challita, W. Saad, C. Yin, and M. Debbah, "Artificial neural networks-based machine learning for wireless networks: A tutorial," IEEE Communications Surveys \& Tutorials, vol. 21, no. 4, pp. 30393071, 2019.

[13] Y. Sun, Y. Wang, J. Jiao, S. Wu, and Q. Zhang, "Deep learning-based long-term power allocation scheme for NOMA downlink system in SIoT," IEEE Access, vol. 7, pp. 86288-86296, 2019.

[14] L. Lei, E. Lagunas, Y. Yuan, M. G. Kibria, S. Chatzinotas, and B. Ottersten, "Beam illumination pattern design in satellite networks: Learning and optimization for efficient beam hopping," IEEE Access, vol. 8, pp. 136655-136667, 2020 .

[15] Y. Zhang, Z. Wang, Y. Huang, J. Ren, Y. Yin, Y. Liu, G. F. Pedersen, and M. Shen, "Deep neural network-based receiver for next-generation LEO satellite communications," IEEE Access, vol. 8, pp. 222 109-222 116, 2020.

[16] A. Wang, L. Lei, E. Lagunas, S. Chatzinotas, and B. Ottersten, "Completion time minimization in NOMA systems: Learning for combinatorial optimization," IEEE Networking Letters, vol. 3, no. 1, pp. 15-18, 2021.

[17] S. Boyd and L. Vandenberghe, Convex optimization. Cambridge university press, 2004.

[18] A. Vinel and P. A. Krokhmal, "Mixed integer programming with a class of nonlinear convex constraints," Discrete Optimization, vol. 24, pp. 6686, 2017.

[19] M. Lubin, E. Yamangil, R. Bent, and J. P. Vielma, "Extended formulations in mixed-integer convex programming," in International Conference on Integer Programming and Combinatorial Optimization. Springer, 2016, pp. 102-113.

[20] S. Wiese, "The mixed-integer conic optimizer in Mosek," https://docs. mosek.com/slides/2018/ismp2018/ismp-wiese.pdf, July 2, 2018.

[21] I. Goodfellow, Y. Bengio, and A. Courville, Deep learning. MIT press, 2016.

[22] ESA, "SATellite Network of EXperts (SATNEX) IV," https://satnex4 org/.

[23] M. S. Ali, H. Tabassum, and E. Hossain, "Dynamic user clustering and power allocation for uplink and downlink non-orthogonal multiple access (NOMA) systems," IEEE access, vol. 4, pp. 6325-6343, 2016.

[24] B. Di, L. Song, and Y. Li, "Sub-channel assignment, power allocation, and user scheduling for non-orthogonal multiple access networks," IEEE Transactions on Wireless Communications, vol. 15, no. 11, pp. 76867698, 2016. 